\title{
A Simple and Compliant Force Sensing Palm for the MLab Simple Hand
}

\author{
Garth Zeglin, Alberto Rodriguez and Matthew T. Mason \\ The Robotics Institute - Carnegie Mellon University \\ garthz@cmu.edu, albertor@cmu. edu, matt.mason@cs.cmu.edu
}

\begin{abstract}
Sensing the forces applied to the palm of a robot manipulator requires robust compliant sensors guarded against collision. The force sensing palm for the MLab Simple Hand measures three components of net contact force in a simple robust design using flexure springs and optical position sensing. The hand is calibrated in an automatic process. Measurements are included to compare performance to the theoretical model.
\end{abstract}

\section{INTRODUCTION}

Force and tactile sensors are used in robot manipulators to control contact force, detect collisions, estimate object pose, identify material properties, and recognize objects. This work focuses on sensing contact force on the "palm" of a manipulator.

Our working hypothesis is that measuring any components of the force and torque applied to the palm by an object during a hand-centric grasp [4] will help disambiguate object pose. In this scenario, force sensing is a low-bandwidth means of perception rather than a high-bandwidth feedback signal. This paper presents a robust and compliant palm contact sensor designed for this application. Our goal is a repeatable measure of quasi-static forces to use in statistical modeling of the grasp process.

Conventional force and torque sensors are engineered for stiffness and high bandwidth. These properties are important for direct force control. For manipulators the stiffness is problematic since it creates high forces during routine collisions with objects and the environment. We have instead chosen to use softer contact which trades off bandwidth for resilience.

Our prototype is an inexpensive compliant robot palm with integral three-axis force sensing. It uses flat steel flexure springs for repeatability and low backlash. It senses normal force and two torque components using inexpensive optical sensors. The paper is intended to explain the rationale and validate that the prototype produces meaningful force and torque data. This is still an early validation of the concept and there are opportunities to optimize the design and improve precision.

\section{A. Simple Hands Project}

We loosely define the "palm" as the unactuated grasp contact surface between active finger elements. The palmar

This work was supported by National Science Foundation [NSF-IIS0916557]. This work does not necessarily reflect the position or the policy of the U.S. Government. No official endorsement should be inferred.

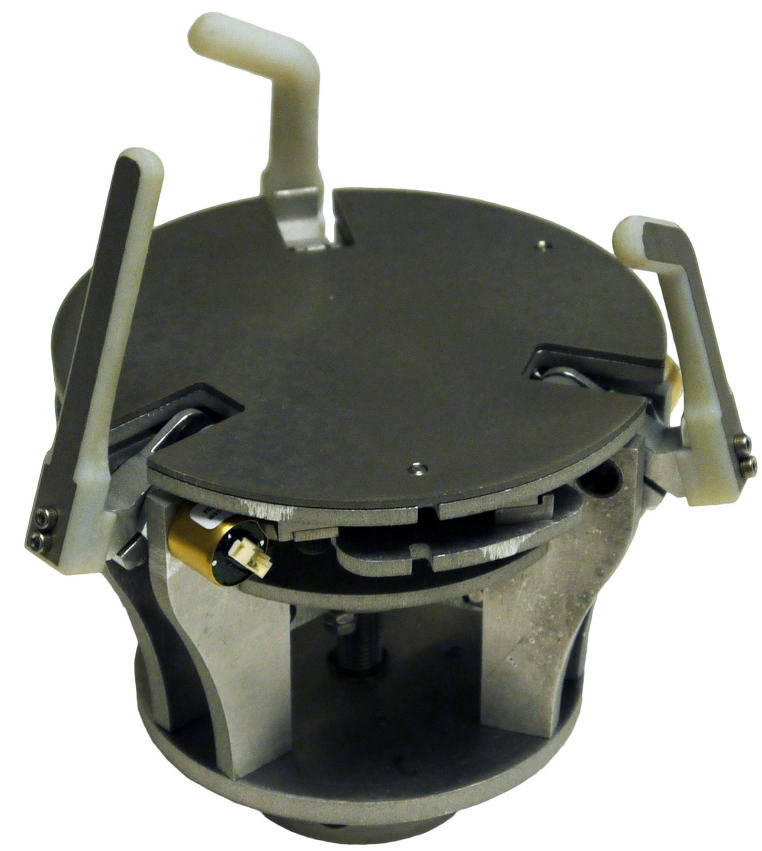

Fig. 1. Force Sensing Palm installed on the MLAB P3 Simple Hand. The circular outer edge of the palm is $101.6 \mathrm{~mm}$ in diameter.

surface is an essential part of many human hand grasps [5] and central to the theory of the Simple Hands Project [8], which aims at building a simple gripper capable of generalpurpose autonomous manipulation.

The central idea is to develop a hand-centric grasp process, where both fingers and palm "push" the object into a stable configuration. The MLab hand has a flat low-friction palm and three fingers compliantly coupled to a single actuator. Grasping produces a small number of stable poses for an object.

We learn data-driven models of the haptic sensing for inhand object recognition and localization. This makes sensor reliability a key driving factor in the design of the MLab hand. This paper describes the development of the robust low-cost palm sensor used by the MLab hand.

\section{B. Stiff Force Sensing}

The principle behind all force and torque sensors is to arrange precision springs with displacement sensors to measure a basis of strain modes resulting from an applied 
force. The springs are typically stiff to minimize deflection and keep resonant frequencies high for high force measuring bandwidth. The small deflections minimize kinematic changes and allows the sensor to be treated as a rigid body rather than a joint.

The stiffness comes at a cost by requiring high-gain amplification and reducing the effectiveness of limit stops. The small displacements require the use of strain gages or piezoelectric sensors. These are not intrinsically expensive, but they do require stable high-gain differential amplification and thermal compensation, raising the complexity of the electronics.

All springs deform plastically above their limits, so all force sensors require protection if exposed to unbounded collision forces. Limit stops add a non-linear stiffness transition to limit deformation. High-stiffness force sensors require the stops to operate over very small displacements and be exceptionally stiff.

If an application can tolerate kinematic change and lower bandwidth, softer force sensors can mitigate collisions, reduce electronics complexity, and simplify overload protection. The sensor displacements become macroscopic and can be sensed with low-cost electronics. The limit stop design becomes easy since the natural structural stiffness is much higher than the elastic elements.

The primary disadvantage of low stiffness is the low bandwidth of dynamic sensing, with a proportionally lower resonant frequency and longer transient response. This is not a problem for our expected applications as long as transients still decay faster than the movement and sensing cycle.

\section{Palm Working Principles}

The palm itself is a smooth rigid plate suspended on three springs, as illustrated in Figure 2. This structure displaces in response to normal force $F_{z}$ and moments $T_{x}$ and $T_{y}$ around axes in the plane of the palm. The compliance structure uses three flat flexure springs. This allows the three degrees of freedom while providing rigidity to the tangential forces $F_{x}$ and $F_{y}$ and normal twisting moment $F_{z}$. The off-axis components produce minimal deflection since they load the flat springs in axial tension and compression and in shear across their wide axes, i.e., the directions in which they are quite stiff. The three components of deflection are sensed using three inexpensive optical transmissive photosensors by modulating light intensity with a moving film printed with a gradient pattern.

This morphology was motivated by the hand-centric design of the smooth palm. During grasps, objects slide and rotate across the palm, and so the tangential forces and twisting moment on the palm are low. The compliant structure design offers relatively rigid support for these forces and torques, and the optical sensors are insensitive in these directions.

\section{PROTOTYPE IMPLEMENTATION}

This section describes our specific prototype as a prelude to the mechanical analysis in Section III. Figure 1 shows the force sensing palm installed on the MLAB Prototype 3

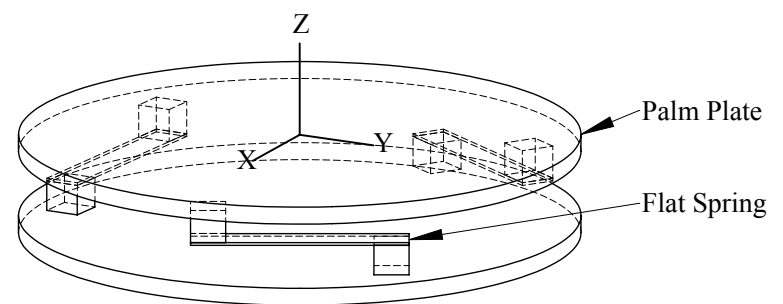

Fig. 2. Simplified diagram of the palm structure showing the palm plate suspended on three flat springs.

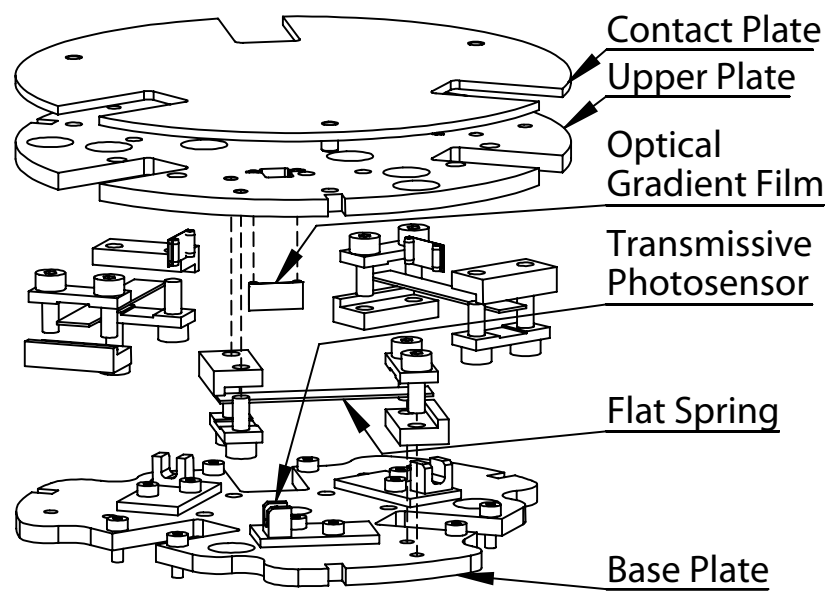

Fig. 3. Exploded view of force sensing palm, showing the attachment of the flat springs to the floating upper assembly and fixed base plate.

Simple Hand. Figure 3 shows an exploded view of the palm assembly alone, and Figure 4 illustrates the location of the essential components from a top perspective.

The exposed surface is the polished steel "Contact Plate." It is rigidly attached to the "Upper Plate" and this structure is suspended above the "Base Plate" on three flat springs. At the compression limit the spring mounts collide with the base plate to directly transmit contact forces into the hand structure and prevent spring damage.

The deflection of the palm structure is measured by three optical transmissive photosensors mounted on circuit boards attached to the base plate. These sensors each incorporate an infrared LED and phototransistor in a single housing. Each beam is modulated by a pattern on a piece of film glued to a slot in the upper plate. The pattern is chosen to be only sensitive to movements along the hand axis.

\section{Mechanical Properties}

This section develops the mechanics of the sensing process and the contact stiffness function. Notably, the stiffness of the assembly varies radially with contact. The stiffest point is at the center when all three springs are equally engaged, and drops off radially toward the edges. This analysis will be used to justify the calibration procedure discussed in Section V.

\section{A. Assumptions and Variables}

The following analysis assumes that a single contact is made with the palm surface exerting a pure normal force 


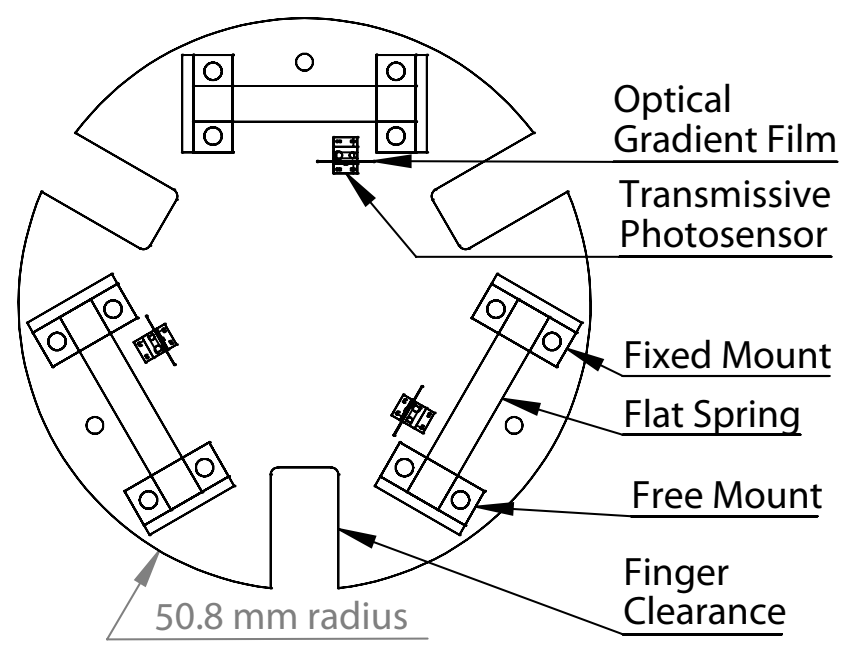

Fig. 4. Placement of essential palm components as viewed from above. The optical gradient films move with the upper structure in response to contact forces.

$F_{z}$ at $\left(x_{f}, y_{f}\right)$, the location of the single contact in palm coordinates. The origin is defined to be the center of the palm surface in the undeflected state. Negative $F_{z}$ values represent forces which compress the palm.

The rotation of the palm is assumed to be negligible for the purposes of determining force directions. The front surface is assumed to remain planar and be described by a height field $\Delta z(x, y)$, where a negative displacement represents compression of the palm surface.

The flat suspension springs are modeled as ideal linear springs attached at three points at radius $R$ from the palm center with equal angular spacing. The spring locations can be specified as vectors $R_{1}, R_{2}, R_{3}$ in the palm coordinates.

$K$ is the stiffness of a single spring in the $\mathrm{Z}$ direction. The three suspension springs apply reaction forces $\left(F_{1}, F_{2}, F_{3}\right)$ upwards to the palm. These are also assumed to lie along $\mathrm{Z}$.

The analysis is quasi-static and neglects the palm mass.

\section{B. Single Contact Stiffness}

The stiffness of the palm for a single contact exerting a normal force varies with location, as illustrated by the fitted curve in Figure 8 . The function is radially symmetric with the following form:

$$
k(x, y)=\frac{3 K R^{2}}{2 x^{2}+2 y^{2}+R^{2}}
$$

This can be derived algebraically by constructing the force and torque equilibrium of the applied force and the three spring reaction forces:

$$
\begin{gathered}
F_{1}+F_{2}+F_{3}+F_{z}=0 \\
\left(R_{1} \times F_{1}\right)+\left(R_{2} \times F_{2}\right)+\left(R_{3} \times F_{3}\right)+\left[x_{f}, y_{f}\right] \times F_{z}=0
\end{gathered}
$$

If the springs are equally spaced at a constant radius $R$, and the orientation of the coordinates are chosen such that $R_{1}$ lies on the $X$ axis at $(R, 0)$, the spring forces are as follows:

\begin{tabular}{|c|c|l|}
\hline Quantity & Variable & Value \\
\hline maximum deflection & $\Delta z_{\max }$ & $1.5 \mathrm{~mm}$ \\
maximum force & $F_{\max }$ & $15 \mathrm{~N}$ \\
spring steel modulus & $E$ & $200 \cdot 10^{9} \mathrm{~Pa}$ \\
yield strength & $\sigma_{y}$ & $1014 \cdot 10^{6} \mathrm{~Pa}$ \\
free length & $L$ & $25.5 \mathrm{~mm}$ \\
width & $b$ & $6.35 \mathrm{~mm}$ \\
thickness & $d$ & $0.508 \mathrm{~mm}$ \\
\hline
\end{tabular}

TABLE I

Flat SPRing PARAmeters

$$
\left[\begin{array}{c}
F_{1} \\
F_{2} \\
F_{3}
\end{array}\right]=-\frac{F_{z}}{3 R}\left[\begin{array}{c}
R+2 x_{f} \\
\left(R-x_{f}+\sqrt{3} y_{f}\right) \\
\left(R-x_{f}-\sqrt{3} y_{f}\right)
\end{array}\right]
$$

Each individual spring follows a linear constitutive relation $F_{n}=K \Delta z_{n}$. Applying this relation to find the spring displacements and solving for the palm plane yields the height function $\Delta z(x, y)$ as a function of the applied force:

$$
\Delta z(x, y)=\frac{F_{z}\left(R^{2}+2 x_{f} x+2 y_{f} y\right)}{3 K R^{2}}
$$

The stiffness at the point of contact is determined by the ratio $F_{z} / \Delta z$. The displacement at the contact point is thus:

$$
\Delta z=\frac{F_{z}\left(R^{2}+2 x^{2}+2 y^{2}\right)}{3 K R^{2}}
$$

Dividing $F_{z}$ by this quantity yields the stiffness function(1).

\section{Design Details}

This section provides further details on the design rationale behind the prototype palm sensor.

\section{A. Springs}

Each spring is a flat rectilinear steel beam clamped in a mount at both ends. The "Fixed Mounts" each attach a spring end down to the base plate, and the "Free Mounts" each attach an opposite end to the upper plate. The maximum linear vertical travel is $1.5 \mathrm{~mm}$. The total suspended mass is 167 grams.

The design parameters for the flat suspension springs are shown in Table I. The peak force was chosen based on experience with earlier versions of the hand. The peak deflection was chosen based on the size of the transmissive photosensors and a desire to keep the overall height of the device small. From these were developed the specified solution for spring size and properties.

The area moment $I$ of a beam with rectangular crosssection is $b d^{3} / 12$, with a section modulus $Z=b d^{2} / 6$. The spring closely approximates a beam which is fixed at one end and guided at the other, since the clamp on each end constrains the bending to an " $\mathrm{S}$ " shape. This condition follows the relation $\Delta z=F L^{3} /(12 E I)$. For the maximum displacement $\Delta z_{\max }$, the calculated maximum force $F_{\max }$ is equal to $15.06 \mathrm{~N}$, very close to the design goal. In this condition, the stress $\sigma=F L /(2 Z)$ is $7.03 \cdot 10^{8} \mathrm{~Pa}$, so the 


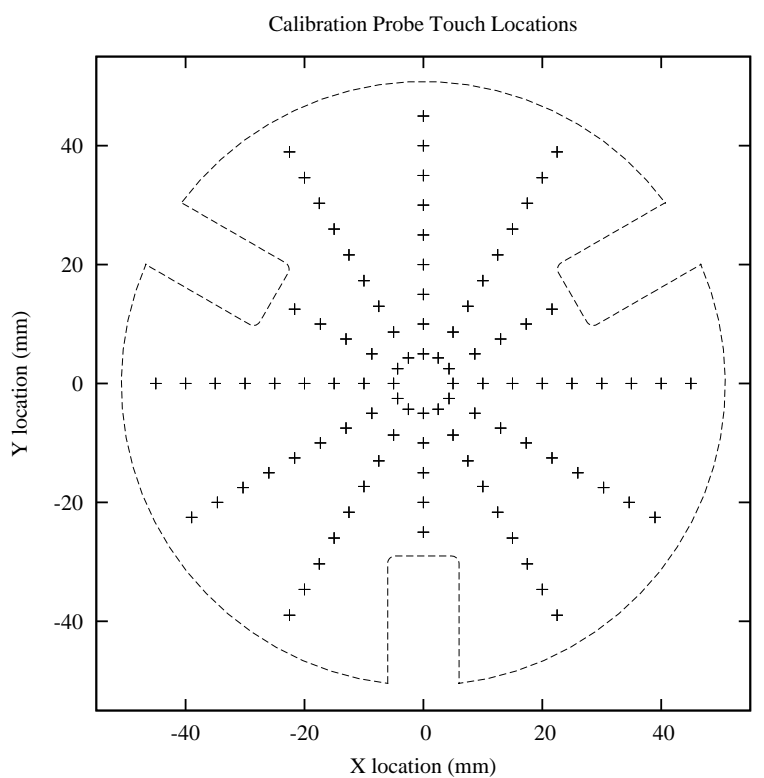

Fig. 5. Location of force calibration probe points. Each point is deflected to three depths by the reference force sensor.

safety factor $\sigma_{y} / \sigma_{\max }$ is 1.44 , i.e., the peak stress is $69 \%$ of the yield stress.

This solution keeps the spring to a manageable length but requires the use of a material with high yield stress. We chose a spring-tempered type 301 stainless steel with a rated yield strength of 147000 psi.

\section{B. Optical Sensors}

The photointerruptors are typically used as binary flag sensors, but if biased correctly have a linear range in which the output signal is a monotonic function of the attenuation of the light between the emitter and receiver.

The film is ordinary transparency film laser-printed with a linear gradient which varies in the vertical direction. The resulting attenuation of the beam results from the absorption of the varying gradient density as integrated over the beam width, and thus linearly varies as the film is moved vertically through the sensor. The film is uniform in the horizontal direction so the sensor is insensitive to other translations. The sensor circuit board incorporates a buffering amplifier with simple offset and gain control and outputs an analog voltage to the hand controller board.

\section{Calibration}

The raw photosensor outputs must be mapped to ground truth contact force in order to provide accurate force measurements. The analog processes in this function include the spring function, the gradient film pattern, the photosensor response, the gain and offset circuit, and the analog-todigital conversion. Our calibration process measures the raw response to known inputs to compute a single composite calibration function combining all effects.

The palm is calibrated using a procedure in which the palm is moved by the robot arm against a reference force sensor ${ }^{1}$ mounted to the robot table. Point contacts are made with a $5 \mathrm{~mm}$ ruby ball probe at a grid of locations as shown in Figure 5, and the normal force $F_{z}$ read from the reference sensor for each of three vertical displacements. The palm has a total travel of $1.5 \mathrm{~mm}$ vertically, so several load conditions can be evaluated at each point by varying the depth of the motion. The uncalibrated palm displacement sensor values are simultaneous read from the palm.

The convenience of this setup is that the same robot arm normally used to operate the hand can be used to measure the palm properties. This allows checking the operation of the palm in the course of normal experiments.

The ABB arm has a specified precision of $0.3 \mathrm{~mm}$. This is more than adequate for varying calibration contact locations over the palm, but the vertical travel of the palm is only $1.5 \mathrm{~mm}$, so the arm precision limits the precision of the applied contact force. This does not pose a problem for the basic calibration of the sensors since the applied force is directly measured using the reference force sensor. It does limit the precision of the stiffness estimation, since the ground truth of vertical displacement is not independently measured.

\section{A. Sensor Calibration}

In quasi-static equilibrium the suspension spring forces balance the applied force as expressed in (2) and (3). The applied force can be rewritten as a force and torque applied to the palm:

$$
T_{x}=y F_{z}, \quad T_{y}=-x F_{z}
$$

In this form the equilibrium equations define a linear relationship between $\left(F_{z}, T_{x}, T_{y}\right)$ and the spring forces $F_{n}$. The individual spring displacement sensors are not separately calibrated, but instead assumed to follow a linear sensor relationship $F_{n}=A S_{n}+B$. The total calibration is lumped into a single linear relationship between uncalibrated values and applied force and torque.

$$
\left[\begin{array}{lll}
F_{z} & T_{x} & T_{y}
\end{array}\right]^{T}=C\left[\begin{array}{llll}
S_{1} & S_{2} & S_{3} & 1
\end{array}\right]^{T}
$$

The sensor vector has been augmented to combine the offset and scale terms into a single $3 \times 4$ calibration matrix $C$. The results of performing this fit on a typical calibration data set are shown in Figure 6. In each plot the force and torque values computed using the calibration matrix and data are plotted against the ground truth values to illustrate the error in the linear fit.

\section{B. Contact Location}

The location of a single contact point can be inferred from the measured force and torque as follows:

$$
x=-T_{y} / F_{z}, \quad y=T_{x} / F_{z}
$$

This is only well-defined for $\left|F_{z}\right|>0$. Figure 7 illustrates the error in reconstructing the contact locations from the calibration set.

\footnotetext{
${ }^{1}$ six-axis ATI mini-40 SI-20-1
} 

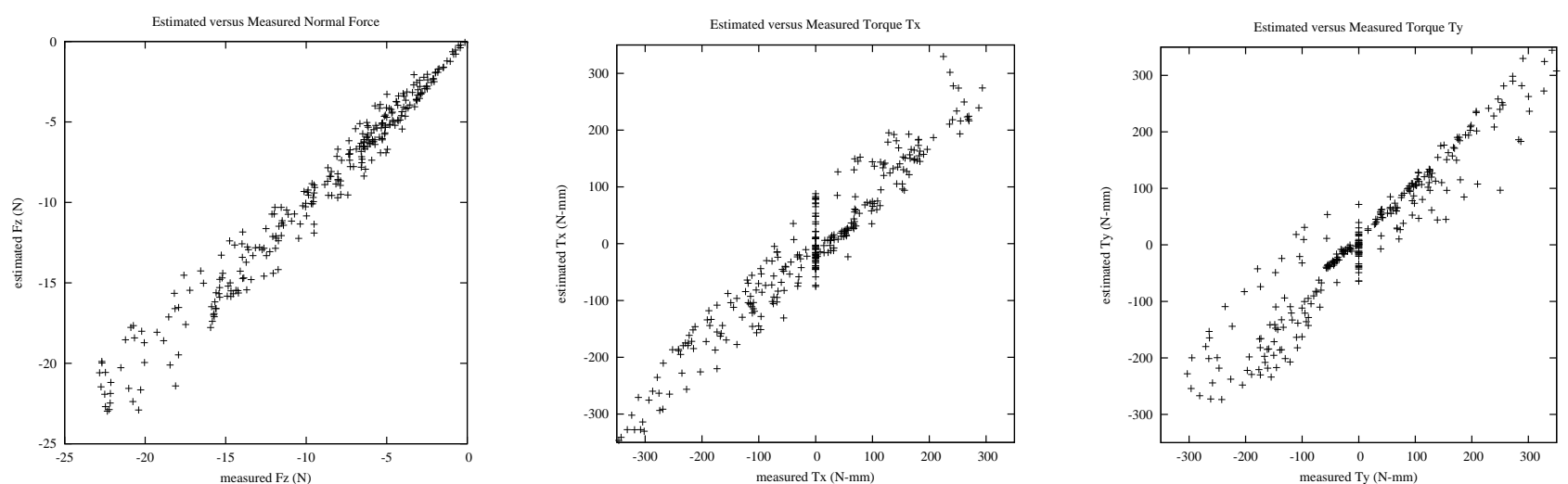

Fig. 6. Force and moment residual calibration error scatter plots. The points would ideally form a diagonal line. Normal force $F_{z}$ (left) fit error has a standard deviation of $1.04 \mathrm{~N}$ with zero mean. Moment $T_{x}$ (middle) fit error has a standard deviation of $39.2 \mathrm{~N}$-mm with zero mean. Moment $T_{y}$ (right) fit error has a standard deviation of $40.9 \mathrm{~N}$-mm with zero mean.
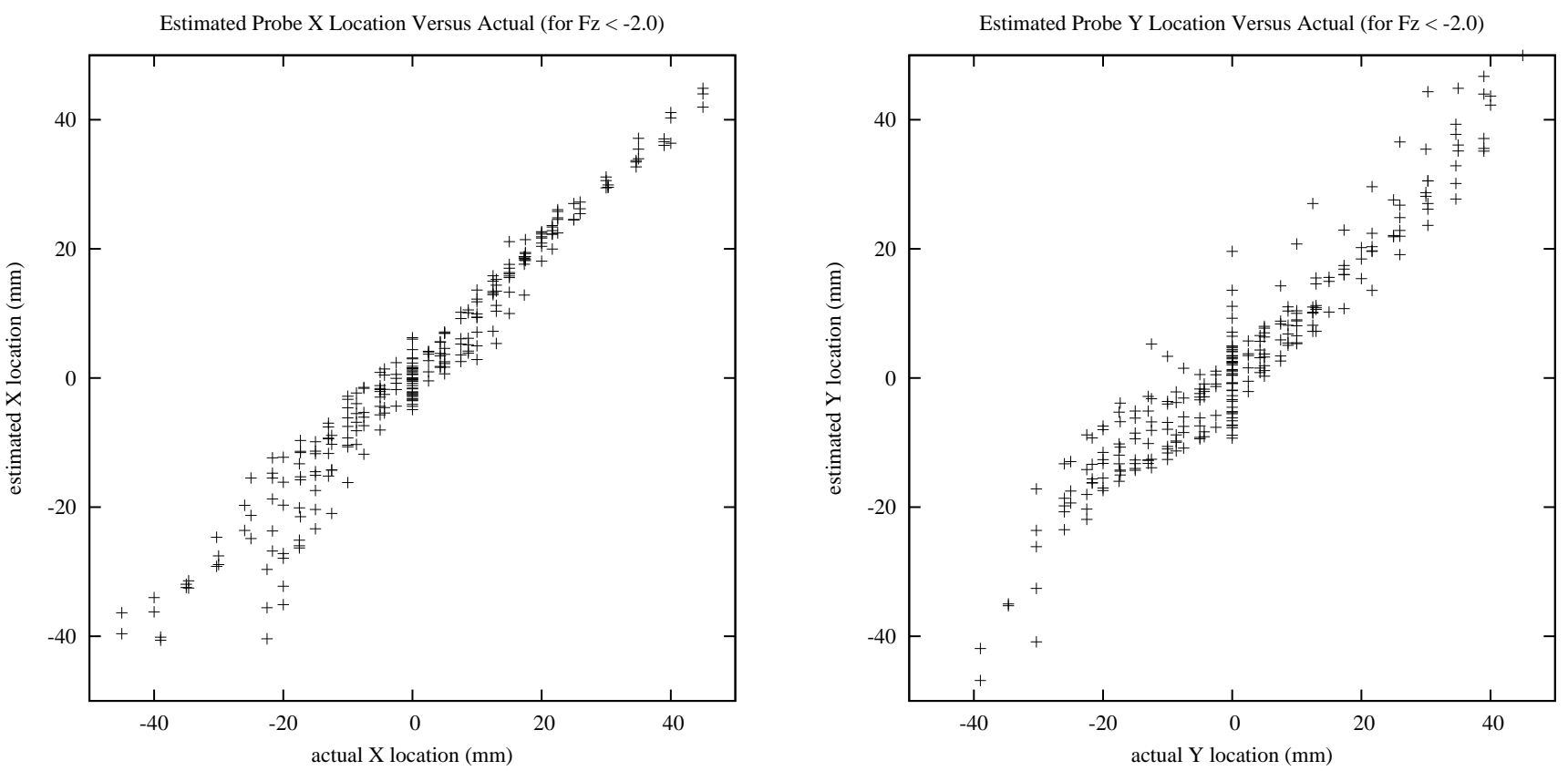

Fig. 7. The position error in the contact locations reconstructed from the calibration set. The $\mathrm{X}$ error (left) has standard deviation of $4.3 \mathrm{~mm}$ with $0.18 \mathrm{~mm}$ mean. The Y error (right) has standard deviation of $7.6 \mathrm{~mm}$ with $1.0 \mathrm{~mm}$ mean.

\section{Vi. Mechanical Evaluation}

This section presents additional mechanical and performance measurements on the prototype.

\section{A. Measured Spring Response}

The spring function can also be estimated from the contact data. This is not essential for normal operation, but helps validate the performance of the mechanical design. This stage of calibration estimates the contact stiffness function $k(x, y)$ from the set of raw data tuples $\left(x_{f}, y_{f}, z_{f}, F_{z}\right)$.

The angular error of the arm kinematics is estimated by processing the arm and force sensor trajectory to extract the initial contact locations during the slow approach of the palm to the sensor. Fitting a plane to these points produces a slight correction for the a priori contact depths.
The spring function is assumed to be radial, parameterized by $K$ and $R$. A linear least-squared fit is performed by optimizing these parameters to minimize the following expression derived from (5):

$$
\left|2 F_{z}\left(x_{f}^{2}+y_{f}^{2}\right)+F_{z} R^{2}-z_{f} 3 K R^{2}\right|
$$

The result of the fit is shown in Figure 8, with values estimated at $K=9.07 \mathrm{~N} / \mathrm{mm}$ and $R=37.7 \mathrm{~mm}$. The spring constant is close to the design value of $10 \mathrm{~N} / \mathrm{mm}$. The radial position of the springs is close to the actual location of the flat springs, even though it based on the simplified point spring model.

\section{B. Self-Response to Gravitational Forces}

The calibration of the palm is performed with the palm facing straight down since that is the expected mode of use. 


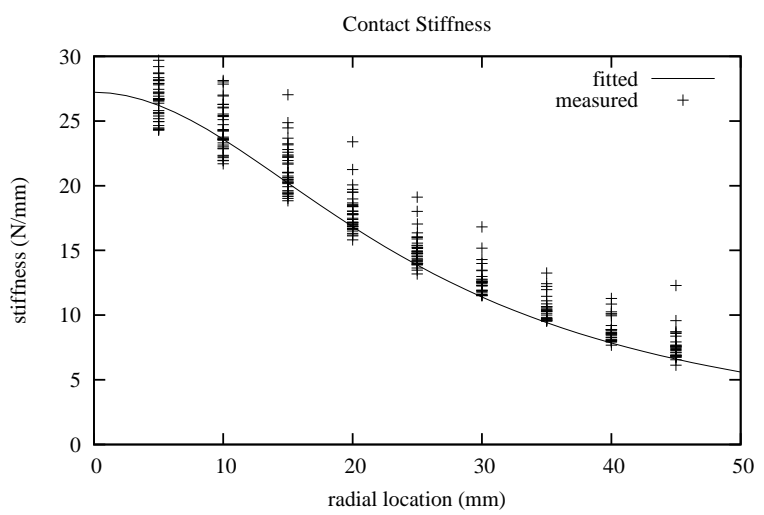

Fig. 8. Estimated stiffness versus radius of contact from palm center, showing the calibration measurements and fitted result. The fit error has a standard deviation of $1.4 \mathrm{~N} / \mathrm{mm}$ and mean of $1.0 \mathrm{~N} / \mathrm{mm}$.

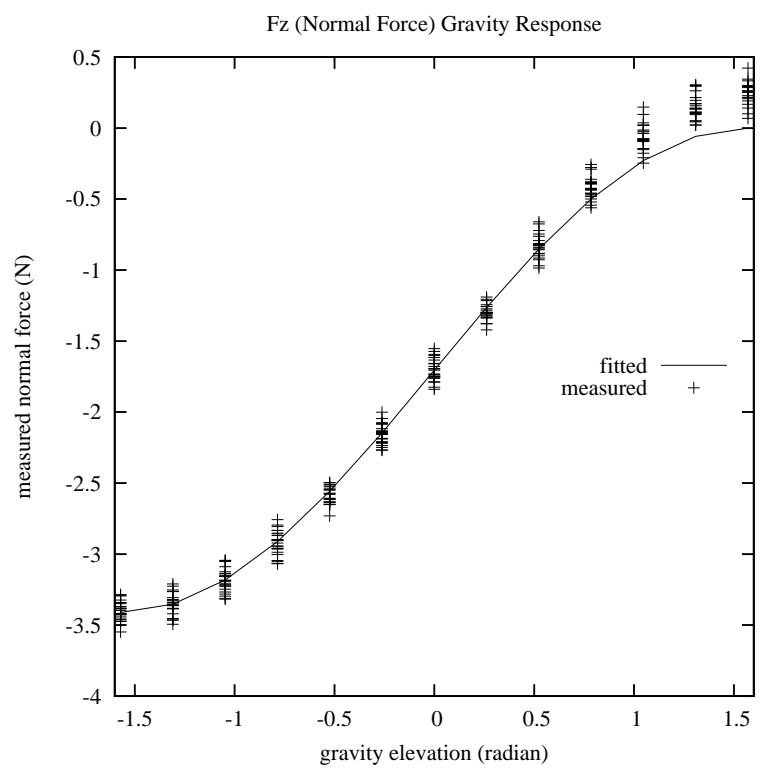

Fig. 9. The plot shows the gravity-induced normal force versus elevation angle. The gravity vectors were sampled over a sphere. The measured value is close to zero at $\pi / 2$ corresponding to the calibration pose with the palm facing down. The fit error has a standard deviation of $0.12 \mathrm{~N}$ and mean of $-0.05 \mathrm{~N}$.

However, the center of the suspended mass is offset $5.5 \mathrm{~mm}$ from the center of rotation of the springs and does respond to gravity as it is reoriented. This response is evaluated using a test procedure which systematically moves the palm over a set of orientations spanning a sphere and while measuring the unloaded response.

Assume that the mass $m$ is displaced from the rotational center by $r_{m}$. The gravity vector with positive magnitude $m g$ is defined by azimuth $\phi$ and elevation $\theta$ such that the gravity force in palm coordinates is as follows:

$$
G=m g[-\cos \theta \sin \phi \quad-\cos \theta \cos \phi \quad \sin \theta]
$$

The moment of the palm gravity force resulting from a given orientation is $r_{m} \times G$ and the normal force is $m g \sin \theta$. Since the palm is calibrated face down, the measured values have offsets in $F_{z}$ of $m g$ and in moment of $r_{m} \times m g z$.
The gravity was sampled on 15 degree intervals in both $\phi$ and $\theta$ (i.e. a non-uniform sampling on a full sphere). Assuming a nominal gravity of $9.81 \mathrm{~m} / \mathrm{s}^{2}$, a least-squares fit of the measured $F_{z}$ yields an estimated mass of 174 grams, close to the measured value of $167 \mathrm{~g}$, and the fitted response curve shown in Figure 9.

\section{Palm Structural Resonance}

The palm is a mass suspended on three springs. The simplest, purely vertical oscillation mode is determined by the suspended mass and the combined spring stiffness with frequency $\sqrt{3 K / M} /(2 \pi)$, where $3 K$ is the combined stiffness of the three springs in parallel. The suspended mass is $0.167 \mathrm{~kg}$ and the computed individual stiffness is $9070 \mathrm{~N} / \mathrm{m}$, so the resonance is expected to be at $64 \mathrm{~Hz}$. This mode was measured using a microphone to record the audible vibration resulting from tapping the palm center. The dominant measured frequency was $65 \mathrm{~Hz}$ with a decay time constant of $67 \mathrm{msec}$.

This vibration rate is about twice as high as the sampling rate currently measured by the Arduino microcontroller on the hand controller, e.g., the sampling rate is approximately one quarter of the Nyquist limit. For this reason, the current system is unsuitable for any dynamic measurement involving substantial transients. To eliminate transients during calibration, the arm movements are slow and the system is allowed to settle for $500 \mathrm{msec}$ prior to reading the sensors.

\section{RELATED WORK}

This paper builds upon the work of the Simple Hand project[8][13]. The demonstration of probabilistic grasp strategies using sensor-based abort and retry [14] and statistical grasp modeling [9] are direct motivation for extending the sensor suite of the Simple Hand prototypes with palm sensing to improve grasp modeling.

This palm design implements a solution to the threeaxis planar case of the more general problem of "intrinsic contact sensing"[1]. The basic process is similar to a patented three-axis planar device which senses force magnitude and location[11]. In both cases a primary difference is the assumption of soft elasticity and macroscopic movement in this design.

The idea of a robot palm has arisen as a general nonprehensile manipulator[3] but its role as a general purpose manipulator in conjunction with fingers is under-explored.

Optical displacement sensors have been explored in force sensing[7], including the use of inexpensive photointerruptors[6], and in parallel with three axes of microscale flexure bearings[10]. However, these sensors have still been conventionally stiff, and did not use a gradient film to extend the range of the photosensors.

A large body of work in manipulator sensing is concerned with designing sensor arrays to produce a dense image of contact forces on a hand[16], usually motivated by the properties of human tactile sense[2]. This typically involves tradeoffs in robustness, cost, or accuracy to solve the difficult problem of distributing tiny force sensors over a wide area 
and communicating with them[15]. These extrinsic sensing approaches are substantially more complex than the minimal approach of this design, but are essentially orthogonal, since extrinsic sensing could always be added to the palm surface.

\section{DISCUSSION}

This work presents a specific palm device to meet the force sensing needs of this project. It also suggests that any manipulator force sensing application should consider whether intrinsic stiffness is required or desirable. Implicit in the approach is the assumption that a palm surface is an important manipulation resource even if unactuated.

\section{A. Simplicity and Low Cost Design}

A guiding principle of the Simple Hand project is to reduce the complexity of manipulator design by efficiently using sensor data with refined models of the grasp process.

Simplicity frequently implies low-cost, which we believe provides direct benefits to the research community by expanding access to hardware. Low cost as a design constraint also stimulates creative solutions.

Our prototypes are fabricated using rapid prototyping methods with secondary machining. The primary features are initially waterjet-cut with some rough holes drilled and tapped afterwards. Most small parts are inexpensive off-theshelf components. The springs are stock metal cut to length. The spring mounts are currently machined but might be simplified in the future.

This design style emphasizes parts with prismatic 2D contours compatible with waterjet or laser cutting. Given the amount of manual machining, it is not yet low-cost, but reflects a design process directed toward low-cost production.

The most expensive component we use is the calibration force sensor. The gravity response experiment suggests that an offline calibration could instead use much cheaper reference masses attached to the palm, using the robot to reorient the palm to sample gravity force and torque.

\section{B. Future Work}

This is an early prototype device and there are a number of engineering refinements which may improve the accuracy. The suspended mass could be reduced to increase the resonant frequency. The optical gradient film could be optimized for greater signal variation over the actual travel of the palm. A flexible skirt can be added to reduce the effects of stray light.

The smooth palm surface is a general solution for handcentric grasping but the addition of friction and surface features may provide useful opportunities for passive object location. This has the potential to create twist moments and lateral forces which cannot be sensed with this design. One solution might be to integrate a second movement stage to provide compliance and force sensing in the remaining orthogonal freedoms.

Statistical learning methods have been previously demonstrated to extract useful indicators of grasp success from the force signature of a single-axis sensor[12]. This suggests that refining the data processing of this device to improve dynamic measurements could provide useful grasp measurements from continuous force data. The success will depend on properly modeling the natural dynamics to filter the external force signals from the low-frequency natural dynamic responses.

Similarly, in-hand manipulation could take the form of regrasping while an object moves via inertia or gravity over the surface of the palm. This could also be performed quasistatically, but good dynamic force sensing would create opportunities for closed-loop control of active regrasping.

\section{REFERENCES}

[1] A. Bicchi, J. K. Salisbury, and D. L. Brock, "Contact sensing from force measurements," International Journal of Robotics Research, vol. 12, no. 3, pp. 249-262, 1993.

[2] R. S. Dahiya, G. Metta, M. Valle, and G. Sandini, "Tactile sensingfrom humans to humanoids," IEEE Transactions on Robotics, vol. 26, no. 1, February 2010.

[3] M. Erdmann, "An exploration of nonprehensile two-palm manipulation," International Journal of Robotics Research, vol. 17, no. 5, pp. 485-503, 1998.

[4] R. Fearing, "Simplified grasping and manipulation with dextrous robot hands," IEEE Journal of Robotics and Automation, vol. 2, no. 4, pp. 188-195, 1986.

[5] T. Feix, R. Pawlik, H. Schmiedmayer, J. Romero, and D. Kragic, "The generation of a comprehensive grasp taxonomy," in Robotics, Science and Systems Conference: Workshop on Understanding the Human Hand for Advancing Robotic Manipulation, 2009.

[6] G. M. Gu, Y. K. Shin, J. Son, and J. Kim, "Design and characterization of a photo-sensor based force measurement unit (FMU)," Sensors and Actuators A: Physical, vol. 182, pp. 49-56, August 2012.

[7] S. Hirose and K. Yoneda, "Development of optical six-axial force sensor and its signal calibration considering nonlinear interference," in Proceedings of IEEE International Conference on Robotics and Automation, 1990, pp. 46-53.

[8] M. T. Mason, A. Rodriguez, S. S. Srinivasa, and A. S. Vazquez, "Autonomous Manipulation with a General-Purpose Simple Hand," The International Journal of Robotics Research, vol. 31, no. 5, pp. 688-703, 2012.

[9] R. Paolini, A. Rodriguez, S. S. Srinivasa, and M. T. Mason, "A Data-Driven Statistical Framework for Post-Grasp Manipulation," in International Symposium on Experimental Robotics (ISER), 2012.

[10] J. Peirs, J. Clijnen, D. Reynaerts, H. V. Brussel, P. Herijgers, B. Corteville, and S. Boone, "A micro optical force sensor for force feedback during minimally invasive robotic surgery," Sensors and Actuators A: Physical, vol. 115, pp. 447-455, September 2004.

[11] G. Peronneau and H. Poizat, "Position-indicating system," U.S. Patent 3,657,475, filed Mar 17, 1970, Issued Apr 18, 1972. 8 claims.

[12] A. Rodriguez, D. Bourne, M. T. Mason, G. F. Rossano, and J. Wang, "Failure Detection in Assembly : Force Signature Analysis," in IEEE Conference on Automation Science and Engineering (CASE), 2010.

[13] A. Rodriguez, M. T. Mason, and S. S. Srinivasa, "Manipulation Capabilities with Simple Hands," in International Symposium on Experimental Robotics (ISER), 2010.

[14] A. Rodriguez, M. T. Mason, S. S. Srinivasa, M. Bernstein, and A. Zirbel, "Abort and Retry in Grasping," in IEEE International Conference on Intelligent Robots and Systems (IROS), 2011.

[15] A. Schmitz, P. Maiolino, M. Maggiali, L. Natale, G. Cannata, and G. Metta, "Methods and technologies for the implementation of largescale robot tactile sensors," IEEE Transactions on Robotics, vol. 27, no. 3, pp. 389-400, June 2011.

[16] H. Yousef, M. Boukallel, and K. Althoefer, "Tactile sensing for dexterous in-hand manipulation in robotics-a review," Sensors and Actuators A: Physical, vol. 167, no. 2, pp. 171-187, June 2011. 